\title{
Sponsoring effektiv und effizient gestalten
}

Sponsoring ist ein wichtiges Marketing-Instrument. Sein Wertschöpfungsbeitrag ist jedoch nicht auf den ersten Blick sichtbar. Ein professionelles Sponsoring-Controlling hilft, die Wirksamkeit und Wirtschaftlichkeit von Sponsoring-Engagements messbar zu machen. Je nach Phase eines Sponsorships sind dabei unterschiedliche Aspekte zu beachten.

Dominik Schwizer, Sven Reinecke 
Für Unternehmen wird es zunehmend schwieriger, Marketingbotschaften über traditionelle Kommunikationskanäle wie TV-Spots, Inserate oder Plakate zu verbreiten. Um aus der Fülle an Werbung herauszustechen, mit der potenzielle Konsumenten tagtäglich konfrontiert werden, geben Unternehmen immer mehr Geld für Sponsoring-Aktivitäten aus (IEG 2016). Sponsoring hat als Kommunikationsinstrument auch insgesamt an Bedeutung gewonnen.

Um die Effektivität und Effizienz von Sponsoring-Engagements sicherzustellen, ist ein professionelles Sponsoring-Controlling unumgänglich. Es kann schon vor dem Erwerb eines Sponsorships dazu beitragen, dass alle relevanten Abteilungen im Unternehmen Marketingziele definieren, die eindeutig messbar sind. Damit kann es den Erfolg von Sponsoring-Maßnahmen messen und sichtbar machen. Im Folgenden stehen deshalb insbesondere drei Phasen des Sponsorings im Fokus (vergleiche Abbildung 1):

1. Der Erwerb eines Sponsoring-Rechts

2. Die Aktivierung eines Sponsorings

3. Die Implementierung und Optimierung eines Sponsorings

\section{Erwerb eines Sponsoring-Rechts}

Die Grundvoraussetzung für ein erfolgreiches Sponsoring ist die Auswahl des richtigen Sponsoring-Objekts. Beim Erwerb eines Sponsoring-Rechts muss das Unternehmen deshalb sicherstellen, dass die geplanten SponsoringInvestitionen auf den übergeordneten strategischen Unternehmenszielen basieren. Die Passgenauigkeit zwischen Sponsor und Sponsoring-Objekt, der sogenannte Fit, spielt eine wichtige Rolle, wenn es um die Verbesserung des Images und die Steigerung der Bekanntheit eines Unternehmens geht.

So hilft beispielsweise eine hohe Passgenauigkeit (guter Fit) zwischen Sponsor und Sponsoring-Objekt dem sponsernden Unternehmen, sein bestehendes Image zu verbessern, hat gleichzeitig aber kaum positive Auswirkungen auf dessen Bekanntheit (Meffert/Burmann/Kirchgeorg 2012, S. 705). Eine geringe Passgenauigkeit (schlechter Fit) zwischen Sponsor und Sponsoring-Objekt hingegen kann zu einer negativen Veränderung des Images führen, aber gleichzeitig zur Steigerung der Bekanntheit beitragen (Meffert/Burmann/Kirchgeorg 2012, S. 705).

\section{„Die Grundvoraussetzung für ein erfolgreiches Sponsoring ist die Auswahl des richtigen Sponsoring-Objekts."}

Gerade weil der Nutzen eines Sponsorings im Vorfeld eines Engagements in vielen Fällen nur schwer abschätzbar ist, wird die Entscheidung für oder gegen ein Sponsoring oft nicht primär auf Basis rationaler Kriterien, sondern oftmals noch eher zufällig gefällt. So zeigt sich, dass beim Erwerb von Sponsoring-Rechten neben der Passgenauigkeit (Fit) offensichtlich auch andere Faktoren eine Rolle spielen, beispielsweise persönliche Präferenzen der in die Entscheidung involvierten Führungskräfte. Begeistern sich die jewei-

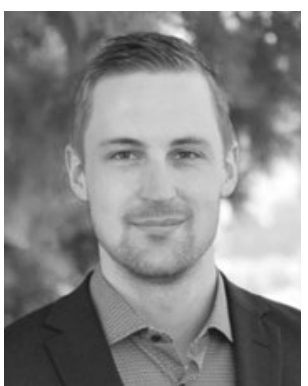

\section{Dominik Schwizer}

ist wissenschaftlicher Mitarbeiter am Institut für Marketing der Universität St. Gallen (HSG).

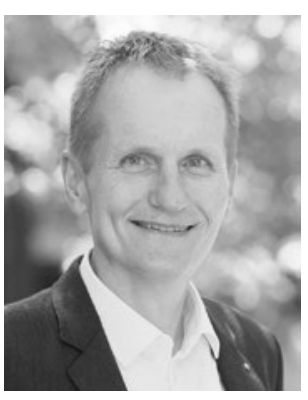

Prof. Dr. Sven Reinecke ist Direktor des Instituts für Marketing an der Universität St. Gallen (HSG).

Dominik Schwizer

Institut für Marketing der Universität St. Gallen (HSG), St. Gallen, Schweiz

E-Mail: dominik.schwizer@unisg.ch

Sven Reinecke

Institut für Marketing der Universität St. Gallen (HSG), St. Gallen, Schweiz

E-Mail: sven.reinecke@unisg.ch 
Investitionsentscheidungen für Sponsorings werden oft noch zufällig getroffen. ligen Verantwortlichen für eine bestimmte Sportart oder einen bestimmten Event besonders, steigt auch die Wahrscheinlichkeit, dass genau dieses Sponsoring-Engagement eingegangen wird. Dies trifft sowohl auf umfangreiche Sponsorships von Großunternehmen als auch auf kleinere SponsoringEngagements mittelständischer Unternehmen zu. Gerade bei weniger umfangreichen Sponsoring-Engagements mit lokaler beziehungsweise regionaler Ausstrahlungskraft beeinflussen bereits bestehende persönliche Kontakte zwischen den Verantwortlichen aufseiten des Gesponserten und des Sponsors die Entscheidung über den Abschluss eines neuen Sponsoring-Vertrags.

\section{„Beim Sponsoring sind nicht nur Investitionen in das Sponsoring-Recht nötig, sondern es bedarf auch genügend Mittel für dessen Aktivierung."}

\section{Konsequenzen für das Sponsoring-Controlling}

Der Auswahl eines Sponsoring-Rechts kommt eine zentrale Bedeutung zu, weshalb die Entscheidung für oder gegen ein Sponsoring-Engagement sorgfältig abgewogen und auf der Basis rationaler Argumente gefällt werden muss. Entscheidend für den Erfolg einer Maßnahme ist die Ansprache der richtigen Zielgruppe. Stehen verschiedene Sponsoring-Engagements zur Auswahl, können beispielsweise Scoring-Modelle dabei helfen, die Attraktivität der einzelnen Sponsorings gezielt zu evaluieren, bevor die entsprechenden Rechte erworben werden. Wichtig ist deshalb, bereits im Vorfeld klare Sponsoring-Ziele für ein mögliches Sponsoring-Engagement festzulegen. Sind die Ziele einmal definiert, können auch die Effizienz und Effektivität der Maßnahmen besser analysiert werden.

\section{Aktivierung eines Sponsorings}

Die Selektion eines passenden Sponsoring-Rechts ist lediglich der erste Schritt in einem über einen längeren Zeitraum andauernden SponsoringProzess. Allein mit der sorgfältigen Auswahl eines Sponsorships ist der Erfolg noch keineswegs sichergestellt, denn ohne Aktivierung bleiben Sponsorships wirkungslos. Daher ist es von entscheidender Bedeutung, wie man die erworbenen Rechte und damit die Verbindung zum Sponsoring-Objekt durch weitergehende Kommunikationsmaßnahmen für die relevante Zielgruppe sichtbar macht. Mit dem Inkrafttreten eines Sponsoring-Vertrags beginnt die Phase der Sponsoring-Aktivierung.

Unter Sponsoring-Aktivierung versteht man diejenigen Investitionen eines Sponsors, die über die Kosten für den Erwerb des Sponsoring-Rechts hinausgehen (vergleiche O’Reilly/Lafrance Horning 2013, S. 424). Die Aktivierungsmaßnahmen sind äußerst vielfältig und reichen von Gewinnspielen über Online-Kampagnen, Mitarbeiterprogramme bis hin zu Direktmarketing und der Kennzeichnung von Merchandising-Artikeln (vergleiche O’Reilly/Lafrance Horning 2013, S. 427). Es geht im Wesentlichen darum, das Sponsoring und die damit verbundene Botschaft bei der anvisierten Ziel- 
gruppe bekannt zu machen. Idealerweise führt dies dazu, dass die Botschaft nicht nur am gesponserten Anlass selbst, sondern auch davor sowie danach weiterverbreitet wird und sich die Wirkung des Sponsorings auf diese Weise zeitlich ausdehnen lässt.

Den Verantwortlichen sollte bewusst sein, dass bei einem Sponsoring nicht nur Investitionen in das Sponsoring-Recht an sich notwendig sind, sondern dass auch genügend Mittel für dessen Aktivierung bereitgestellt werden müssen.

Bei großen Unternehmen ist im Bereich der Sponsoring-Aktivierung eine Professionalisierung festzustellen. Die Herausforderung beim Ergreifen von Aktivierungsmaßnahmen besteht für diese Unternehmen insbesondere darin, die einzelnen Maßnahmen miteinander zu verknüpfen und eine integrierte Kommunikation des Sponsorings nach außen sicherzustellen.

Raiffeisen Schweiz ist ein gutes Beispiel dafür, wie man ein Titelsponsoring auf verschiedenen Kanälen aktivieren und so die Zielgruppe involvieren kann. Das Unternehmen aktiviert sein Sponsorship der Super League, der höchsten Schweizer Fußballliga, mit einer eigens dafür geschaffenen Online-Präsenz. Unter dem Motto „WeLoveFootball.ch“ werden für die Fans auf einer zentralen Plattform Hintergrundinformationen und spannende Geschichten zur Schweizer Fußballmeisterschaft, den involvierten Akteuren und dem aktuellen Spielgeschehen zusammengestellt (Raiffeisen 2016). Anhand der Klickraten und des Surfverhaltens der Nutzer der Website erhält das Unternehmen Anhaltspunkte, welche Inhalte bei der Zielgruppe besonders großen Anklang finden. Dadurch kann Raiffeisen Schweiz ihre Online-Plattform kontinuierlich den Bedürfnissen der Zielgruppe anpassen und noch mehr relevante Inhalte zur Verfügung stellen. Die Ziele dabei sind einerseits, die Fans mit neuen Inhalten zu bedienen, damit sie die Website wiederholt aufrufen, und andererseits, neue Leads zu generieren.

\section{„Das Sponsoring-Controlling übernimmt in der Aktivierungsphase die Aufgabe, die ergriffenen Maßnahmen auf deren Effektivität und Effizienz zu überprüfen."}

Allerdings steht das Unternehmen wie andere Finanzdienstleister und Versicherungen auch vor der Herausforderung, die Konversion von einem reinen Interesse für die Inhalte im Zusammenhang mit Fußball hin zum Kauf eines Produkts oder einer Dienstleistung des Unternehmens sicherzustellen. Dabei muss es äußerst behutsam vorgehen. Würden die Bankprodukte und -dienstleistungen auf der Website zu stark in den Vordergrund gerückt, fühlten sich die Besucher dadurch womöglich gestört und die Attraktivität der Website würde sinken.

\section{Konsequenzen für das Sponsoring-Controlling}

Das Sponsoring-Controlling muss zunächst sicherstellen, dass das Unternehmen von vornherein ein angemessenes Budget für die Aktivierung der

\section{Sponsorships bleiben wirkungslos, wenn ihre Aktivierung vernachlässigt wird.}

\section{Zusammenfassung}

- Sponsoring-Controlling betrifft alle Phasen eines Sponsorings: vom Erwerb eines Sponsoring-Rechts über dessen Aktivierung bis hin zur Implementierung und Optimierung von Sponsoring-Aktivierungsmaßnahmen.

- Voraussetzungen für ein effektives Sponsoring-Controlling sind klar formulierte Zielvorgaben und die Festlegung operationalisierbarer KeyPerformance-Indikatoren.

- Daten für das Sponsoring-Controlling müssen regelmäßig und über einen längeren Zeitraum hinweg erhoben werden, um ein Sponsorship schrittweise zu adaptieren und zu optimieren. 


\section{Sponsoren müssen sich mit
gezielten Aktivierungsmaß-
nahmen von der Konkurrenz \\ Sponsoren müssen sich mit
gezielten Aktivierungsmaß-
nahmen von der Konkurrenz \\ Sponsoren müssen sich mit
gezielten Aktivierungsmaß-
nahmen von der Konkurrenz differenzieren.}

Sponsoring-Maßnahme einplant. Es übernimmt in der Aktivierungsphase die Aufgabe, die ergriffenen Maßnahmen auf deren Effektivität und Effizienz zu überprüfen. So kann die Aktivierung im weiteren Verlauf des Sponsoring-Engagements optimiert und - wo nötig - angepasst werden.

Der finanzielle und personelle Aufwand für das Anstoßen unternehmensinterner Lernprozesse im Zusammenhang mit einem bestimmten Sponsoring sollten nicht unterschätzt werden, da hierfür in der Regel viele Ressourcen gebunden werden. Eine professionelle Implementierung und kontinuierliche Verbesserung der Maßnahmen zur Aktivierung eines Sponsorings bieten Unternehmen jedoch die Chance, sich von anderen Sponsoren zu differenzieren. Dies ist allerdings nur dann möglich, wenn vorab klare Aktivierungsziele definiert wurden. Für jedes Ziel müssen zudem ein oder mehrere empirisch überprüfbare Key-Performance-Indikatoren festgelegt werden. Nur so ist tatsächlich eine Messung realisierbar. Ein professionelles Sponsoring-Controlling geht jedoch über die Erhebung klassischer Messgrößen der Marktforschung wie Image und Bekanntheit oder die Berechnung von Medienäquivalenzwerten und Tausenderkontaktpreisen hinaus. Wünschenswert ist deshalb, die Aspekte der getroffenen Aktivierungsmaßnahmen ganzheitlich zu erfassen. Das bedeutet, dass nicht versucht werden sollte, den Nutzen eines Sponsorings ausschließlich in der kurzen Frist anhand einfach messbarer Marktforschungsdaten festzustellen. Vielmehr müssen diese Prozesse über einen längeren Zeitraum hinweg angestoßen

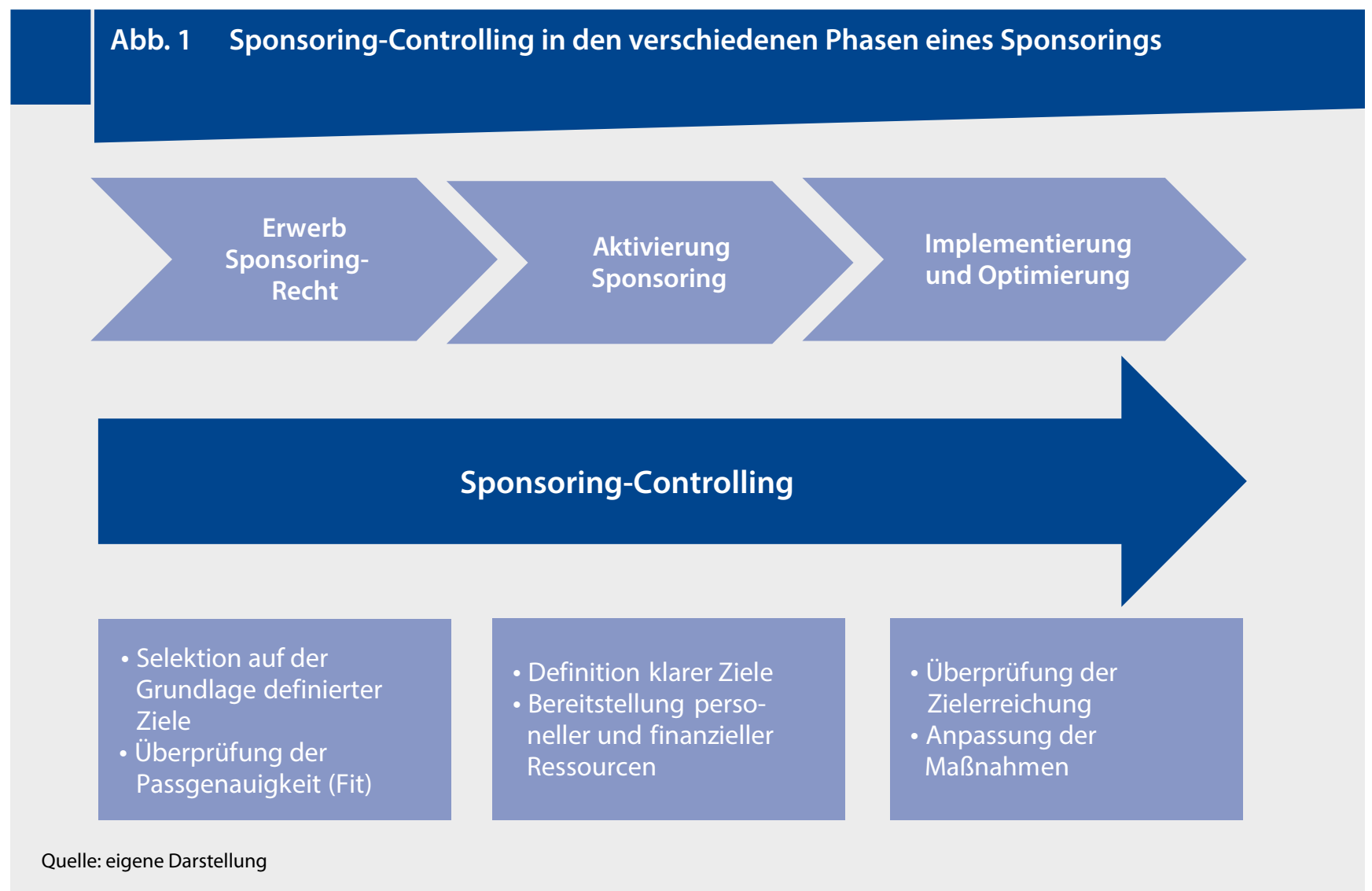


und kontinuierlich verbessert werden. Dazu fehlen bisher jedoch etablierte Standards, da unter anderem die Unternehmen im Bereich der Aktivierungsmaßnahmen meist individuelle, maßgeschneiderte Lösungen entwickeln.

\section{Implementierung und Optimierung}

Das Potenzial von Sponsoring als Kommunikationsinstrument wird häufig noch unterschätzt, was wesentlich damit zusammenhängt, dass sich die Effektivität und Effizienz von Sponsoring nicht auf einfache Art und Weise messen lassen. Erfolge sind zumeist erst langfristig nachzuweisen. Wie bei den meisten Management-Entscheidungen ist aber auch bei Investitionsentscheidungen für Sponsoring-Maßnahmen der Nachweis der Wirksamkeit der getroffenen Maßnahmen von zentraler Bedeutung. Üblicherweise sind die Manager dem Druck ausgesetzt, die Investitionen in Sponsorings in der kurzen Frist rechtfertigen zu müssen. Ideal für sie wäre es deshalb, aufzeigen zu können, dass sich Sponsoring beispielsweise direkt positiv auf die Produktabsätze auswirkt. Dies ist jedoch kaum möglich, da der Verkauf eines Produkts nie ausschließlich auf ein Sponsoring-Engagement zurückgeführt werden kann. Zu viele andere Faktoren beeinflussen die Kaufentscheidungen eines Kunden mit (vergleiche Reinecke/Janz 2007; Rutschmann/Belz 2014).

\section{„Sponsoring basiert auf dem Prinzip von Leistung und Gegenleistung."}

Unternehmen - beispielsweise aus der Automobil- und Finanzindustrie versuchen dennoch, Zusammenhänge zwischen ihren Sponsorships und den Produktabsätzen herzustellen. Doch auch hier zeigt sich, dass Sponsorings ihre Wirkung erst langfristig entfalten. Indem ein Automobilhersteller zum Beispiel seine eingeladenen Gäste mit dem neuesten Modell zum Event abholen lässt, schafft er ein positives Erlebnis in Verbindung mit der eigenen Marke und bringt potenzielle Kunden mit den eigenen Produkten in Kontakt. Die Wirkung solcher Maßnahmen lässt sich allerdings nur indirekt ermitteln. Sponsoring ist deshalb in der Regel als ein Instrument zur Stärkung des Images und zur Erhöhung der Bekanntheit anzusehen und weniger als direktes Verkaufs-Tool (vergleiche Hohenauer 2016, S. 143 f.). Das muss sich auch im Sponsoring-Controlling widerspiegeln. Als Key-Performance-Indikatoren können ausschließlich Kennzahlen herangezogen werden, die sich durch ein Sponsoring-Engagement auch tatsächlich aktiv beeinflussen lassen.

\section{Konsequenzen für das Sponsoring-Controlling}

Sponsoring-Controlling muss langfristig implementiert werden, um die getroffenen Sponsoring-Maßnahmen regelmäßig und über einen längeren Zeitraum evaluieren zu können. Die Wirkung von Sponsoring auf den Absatz von Produkten kann bei der Evaluierung durchaus eine Rolle spielen, sollte aber nicht als alleiniges Kriterium dafür herangezogen werden, ob ein Sponsoring effektiv ist oder nicht. Mehr als solche monetären Sponsoring-Ziele eignet sich die Erreichung psychologischer Ziele, wie etwa die

\section{Sponsoring ist ein Kommu- nikationsinstrument, das seine Wirkung nur in der langen Frist erzielt.}




\section{Durch die zunehmende}

Professionalisierung im

Sponsoring gewinnt das

Controlling an Bedeutung.

\section{Handlungsempfehlungen}

- Definieren Sie vor dem Eingehen eines Sponsoring-Engagements Ihre Sponsoring-Ziele genau.

- Stimmen Sie die Sponsoring-Ziele auf die Gesamtunternehmensstrategie ab, überprüfen Sie regelmäßig die Zielerreichung.

- Überlegen Sie, mit welchen Sponsoring-Maßnahmen Sie Ihre Ziele erreichen können.

- Entwickeln Sie klare Bewertungskriterien für die Auswahl von Sponsoring-Engagements und treffen Sie Investitionsentscheidungen auf deren Grundlage.

- Stellen Sie vor der Investition in ein Sponsorship sicher, dass die personellen und finanziellen Ressourcen für dessen Aktivierung über einen längeren Zeitraum zur Verfügung stehen.
Verbesserung des Unternehmensimages und die Steigerung der Bekanntheit, zum Nachverfolgen, da Sponsoring in der Regel auf diese beiden Aspekte einzahlt. Kontinuität ist dabei ein entscheidender Erfolgsfaktor: Entschließt sich ein Unternehmen für ein Sponsoring, dann sollte es dieses über mehrere Jahre hinweg konsequent verfolgen und weiterentwickeln.

Entscheidend ist, dass ein Sponsoring eine gewisse Kontinuität aufweist. Es macht wenig Sinn, eine Veranstaltung nur ein- oder zweimal zu sponsern, denn die Konsumenten benötigen eine gewisse Zeit, bis sie ein Sponsoring-Objekt mit einem Sponsor in Verbindung bringen. Deshalb wirken sich häufige Wechsel zwischen unterschiedlichen Sponsoring-Objekten, wie zum Beispiel unterschiedlichen Sportarten, negativ auf die Effektivität und Effizienz einer Sponsoring-Strategie aus. Bei einer Neuausrichtung des Sponsorings werden immer wieder neue Zielgruppen angesprochen. Deshalb sollte ein Unternehmen besser weniger Sponsorings eingehen, diese dafür über einen längeren Zeitraum verfolgen und professionell aktivieren.

\section{„Sponsoring-Controlling muss langfristig implementiert werden."}

\section{Schlussbetrachtung}

Sponsoring-Verantwortliche sollten bereits in der Entscheidungsphase für ein Sponsoring-Engagement ein Sponsoring-Controlling etablieren, um die hier relevanten Faktoren prüfen zu lassen und die Wirksamkeit (Effektivität) und Wirtschaftlichkeit (Effizienz) der geplanten Maßnahmen zu gewährleisten beziehungsweise zu optimieren.

Sponsoring basiert auf dem Prinzip von Leistung und Gegenleistung. Ein Unternehmen investiert finanzielle, Sach- oder Dienstleistungen in ein Sponsoring-Objekt und erhält im Gegenzug das Recht, kommunikative Maßnahmen im Zusammenhang mit dem Sponsoring-Objekt zu ergreifen. Diese Investitionen müssen dem Unternehmen einen Nutzen stiften. Das Sponsoring-Controlling kann den Wertschöpfungsbeitrag von Sponsoring bewerten und sichtbar machen.

\section{Literatur}

Hohenauer, R. (2016): Sponsoring-Wirkung auf das Kaufverhalten, Wiesbaden.

IEG (2016): IEG Sponsorship Report, http://www.statista.com/statistics/196864/ global-sponsorship-spending-since-2007/ (letzter Abruf: 19.10.2016).

— ${ }^{*}$ Meffert, H./Burmann, C./Kirchgeorg, M. (2012): Marketing: Grundlagen marktorientierter Unternehmensführung, 11. Auflage, Wiesbaden. www.springerprofessional.de/link/4532610

O’Reilly, N./Lafrance Horning, D. (2013): Leveraging Sponsorship: The Activation Ratio, in: Sport Management Review, 16 (4), S. 424-437.

Papadimitriou, D./Apostolopoulou, A. (2009): Olympic Sponsorship Activation and the Creation of Competitive Advantage, in: Journal of Promotion Management, 15 (1/2), S. 90-117. 
Raiffeisen (2016): Raiffeisen Super League Exklusiv, https://www.welovefootball.ch/ de.html (letzter Abruf: 19.10.2016).

Reinecke, S./Janz, S. (2007): Marketingcontrolling: Sicherstellen von Marketingeffektivität und -effizienz, Stuttgart.

Rutschmann, M./Belz, C. (2014): Reales Marketing: Kunden zum Kauf führen. Kaufprozess - Kaufhandlung - Erfolg, Stuttgart.

* Abonnenten von Springer Professional haben kostenfrei Zugriff.

\section{Ergänzende Studientipps}

Reinecke, S./Hohenauer, R. (2012): Mehr Effektivität und Effizienz durch Sponsoringcontrolling, in: Marke 41 (6), S. 8-14.

Reinecke, S./Janz, S./Hohenauer, R. (2016): Controlling der Marketingkommunikation: Zentrale Kennzahlen und ausgewählte Evaluationsverfahren, in: Esch, F.-R./Langner T./Bruhn M. (2016):

Handbuch Controlling der Kommunikation: Grundlagen - Innovative Ansätze - Praktische Umsetzungen, Wiesbaden, S. 3-25. www.springerprofessional.de/link/10908870

Weitere Empfehlungen der Verlagsredaktion aus www.springerprofessional.de zu:

\section{Sponsoring}

Hohenauer, R. (2016): Sponsoring-Wirkung auf das Kaufverhalten, Wiesbaden. www.springerprofessional.de/link/10799994

Bruhn, M. (2016): Einsatz des Sponsoring für die Marketingkommunikation, in: Bruhn, M./Esch, F.-R./Langner, T. (Hrsg.): Handbuch Instrumente der Kommunikation, Grundlagen - Innovative Ansätze - Praktische Umsetzungen, 2., vollständig überarbeitete und erweiterte Auflage Wiesbaden, S. 185-201.

www.springerprofessional.de/link/10908618

\section{Springer Gabler}

Digitale Transformation erfolgreich gestalten

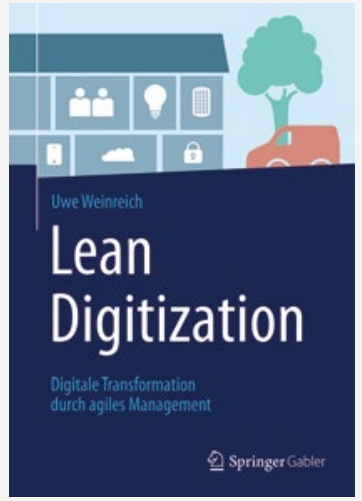

U. Weinreich

Lean Digitization

Digitale Transformation durch agiles Management

2016. X, 285 S. 59 Abb. in Farbe. Geb. $€(D) 39,99 \mid €(A) 41,11$ | ${ }^{*} \mathrm{sFr} 41,50$

ISBN 978-3-662-50501-4

$€ 29,99 \mid{ }^{*} \mathrm{sFr} 33,00$

ISBN 978-3-662-50502-1 (eBook)

- Liefert eine verlässliche, sichere Management Methode, um digitale Transformation ohne Verschwendung und erfolgreich zu gestalten

- Zeigt Vorgehensweisen, wie eine Kultur des kontinuierlichen Lernens im Unternehmen verankert werden kann

$€(D)$ sind gebundene Ladenpreise in Deutschland und enthalten $7 \%$ für Print-produkte bzw. $19 \%$ MwSt. für elektronische Produkte. $€(A)$ sind gebundene Ladenpreise in Österreich und enthalten $10 \%$ für Printprodukte bzw. $20 \%$ MwSt. für elektronische Produkte. Die mit * gekennzeichneten Preise sind unverbindliche Preisempfehlungen und enthalten die landesübliche MwSt. Preisänderungen und Irrtümer vorbehalten.

\section{Part of SPRINGER NATURE}

springer-gabler.de 